\title{
ESTUDO DE REVISÃO DE EFETIVIDADE E SEGURANÇA DOS FÁRMACOS UTILIZADOS NA QUIMIOTERAPIA INTRA-ARTERIAL NO TRATAMENTO DO RETINOBLASTOMA
}

\section{ARTIGO DE REVISÃO}

CANALES, Inácia Maria da Silva ${ }^{1}$

RIBEIRO, Alessandra Maximiano ${ }^{2}$

SOARES, Amata dos Santos ${ }^{3}$

KLINGELBT, Ingrid ${ }^{4}$

ANDREOLLI, Rafael ${ }^{5}$

SANTANA, Claudinei Alves ${ }^{6}$

CANALES, Inácia Maria da Silva. Et al. Estudo de revisão de efetividade e segurança dos fármacos utilizados na Quimioterapia Intra-arterial no

${ }^{1}$ Farmacêutica. Especialista em Farmácia Clínica e Hospitalar (Senac), Graduação em Farmácia e Bioquímica (UNINOVE).

${ }^{2}$ Farmacêutica. Especialista em Farmácia Clínica e Hospitalar (Senac), Graduação em Farmácia (UNIP).

${ }^{3}$ Farmacêutica. Especialista em Farmácia Clínica e Hospitalar (Senac), Graduação em Farmácia (ANHANGUERA).

${ }^{4}$ Farmacêutica. Especialista em Farmácia Clínica e Hospitalar (Senac), Graduação em Farmácia (FOC).

${ }^{5}$ Farmacêutico. Especialista em Farmácia Clínica e Hospitalar (Senac), Graduação em Farmácia (UNIP).

${ }^{6}$ Orientador. Farmacêutico. Mestre em Ciências Médicas, FMUSP. Especialista em Oncologia Multiprofissional (HSL), Especialista em Farmácia Hospitalar (FOC), Graduação em Farmácia Bioquímica (USJT). 
Tratamento do Retinoblastoma. Revista Científica Multidisciplinar Núcleo do Conhecimento. Ano 05, Ed. 10, Vol. 04, pp. 35-51. Outubro de 2020. ISSN: 24480959, Link de acesso: https://www.nucleodoconhecimento.com.br/saude/segurancados-farmacos

\section{RESUMO}

O Retinoblastoma é um tumor maligno característico da infância, o que antes era sinal de enucleação, desde 2005 vem sendo aplicado um tratamento inovador com a quimioterapia intra-arterial que constatou ser efetivo, trazendo bom controle do tumor e complicações sistêmicas mínimas. Pesquisas apresentam resultados de efetividade e segurança desta terapêutica utilizada em alguns países, assim como os principais fármacos utilizados e os resultados positivos no tratamento do retinoblastoma. As informações foram obtidas através de um estudo de revisão na literatura científica, onde se verificou que os fármacos mais utilizados na quimioterapia intra-arterial são o melfalano, a carboplatina e o topotecano. A partir destes resultados podemos concluir que o fármaco de primeira escolha é o melfalano, que pode ser usado em monoterapia ou combinado com carboplatina ou topotecano. Atualmente ainda há estudos em andamento que avaliam a efetividade e segurança do topotecano e da carboplatina na pediatria, sejam em monoterapia ou terapia combinada com outros antineoplásicos.

Palavras-chave: Retinoblastoma, Quimioterapia intra-arterial, melfalano, topotecano, carboplatina.

\section{INTRODUÇÃO}

O Retinoblastoma é uma doença caracterizada por um tumor maligno intraocular originário da membrana neura ectodérmica da retina embrionária, que se manifesta na infância, tendo os sinais e sintomas variando conforme tamanho e localização, sendo o mais comum a leucocoria, também chamada de reflexo do "olho do gato", sendo um critério importante de diagnóstico, podendo ocorrer outras manifestações que incluem cegueira, glaucoma, estrabismo e hiperemia conjuntiva (SELISTRE, 
2013; INSTITUTO ONCOGUIA, 2017; DEPARTAMENTO CIENTÍFICO DE ONCOHEMATOLOGIA, 2015).

Existem duas formas de manifestação da doença, sendo unilateral (cerca de 3 casos em cada 4 crianças) e bilateral (cerca de 1 caso em cada 4 crianças), não havendo uma predisposição de raça e etnias, a ocorrência é quase igualmente em meninos e meninas, podendo afetar igualmente o olho direito ou esquerdo, porém pode ser esporádico ou hereditário (INSTITUTO ONCOGUIA, 2017).

Tendo a porcentagem de 30 a $40 \%$ de origem hereditária com mutação na linha germinativas bilaterais ou multifocais e de 60 a $70 \%$ são formas não hereditárias, e associam-se a mutação somática, unilaterais e unifocais (FABIAN, 2017; PINA, 2012).

A classificação atualmente mais utilizada no estadiamento das formas localizadas deste tipo de tumor é conforme o Sistema de Classificação Internacional do Retinoblastoma Intraocular. Esta classificação baseia-se de acordo com a dimensão da lesão, distância do tumor em relação á fóvea e disco óptico, e presença de disseminação subretiniana ou vítrea (Revista da Sociedade Portuguesa de Oftalmologia).

A classificação internacional do Retinoblastoma intraocular segue apresentada no quadro abaixo:

Quadro 1. Classificação Internacional do Retinoblastoma Intraocular e a Taxa de preservação do globo ocular.

\begin{tabular}{|l|ll|}
\hline $\begin{array}{l}\text { Sistema de Classificação Internacional do } \\
\text { Retinoblastoma Intraocular }\end{array}$ & $\begin{array}{l}\text { Taxa } \\
\text { preservação do } \\
\text { globo ocular na } \\
\text { literatura }\end{array}$ \\
\hline Grupo A: & $100 \%$ \\
\hline $\begin{array}{l}\text { Tumores pequenos (até } 3 \text { mm de diâmetro) confinados à } \\
\text { retina, que não estão perto de estruturas importantes, }\end{array}$ \\
\hline
\end{tabular}


como o disco óptico (local onde entra o nervo óptico) ou do fovéola (centro de visão).

Grupo B:

$93 \%$

Todos os outros tumores (maiores que $3 \mathrm{~mm}$, mas próximos do disco óptico ou fovéola) que ainda estão confinados à retina.

Grupo C:

Tumores bem definidos, com pequena dispersão sob a retina ou para dentro do material gelatinoso que preenche 0 olho.

Grupo D: $47 \%$

Tumores grandes ou mal definidos com humor vítreo comprometido ou acometimento subretiniano. A retina pode se desprender da parte de trás do olho.

Grupo E. $0 \% *$

O tumor é muito grande, se estende até perto da parte frontal do olho, é hemorrágico ou causa glaucoma, ou tem outras características que significam que não há praticamente nenhuma possibilidade do olho poder ser salvo.

Fonte: Adaptado de Instituto Oncoguia (2017).

A incidência dos casos varia de $1 / 16.000$ a $1 / 18.000$ nascidos vivos, afetando 8.102 crianças por ano, em todo o Mundo (NETO, 2016).

No Brasil, a expectativa é de cerca de 400 novos casos por ano, ocorrendo comumente em bebês e crianças pequenas, sendo que a idade média ao diagnóstico é de 2 anos raramente afetando crianças maiores de 6 anos de idade (NETO, 2016; AMERICAN CANCER SOCIETY, 2018). 
Nos Estados Unidos, anualmente 200 a 300 crianças são diagnosticadas com retinoblastoma (AMERICAN CANCER SOCIETY, 2018).

Há 20 anos o tratamento de retinoblastoma era sinônimo de enucleação ou radioterapia externa que na maioria das vezes causava enoftalmia e acentuadas deformidades faciais (RODRIGUES; LATORRE e CAMARGO, 2004; CARDOSO et al., 2011).

Entretanto, as novas técnicas têm mostrado eficiência na regressão tumoral ou pelo menos o controle, com vantagens estéticas e funcionais a partir de técnicas como fotocoagulação a laser, quimioterapia intra-arterial, crioterapia, quimioterapia intravítrea, braquiteria, irradiação por partículas carregadas de prótons ou íons de Hélio e Enucleação (ERWENNE, 1998; AMERICAN CANCER SOCIETY, 2018; INSTITUTO ONCOGUIA, 2018; LIANG, 2020).

Uma forma aplicada atualmente é a Quimioterapia Intra-Arterial desenvolvida nos Estados Unidos em meados de 2005 repercutindo por todo mundo, sendo o Brasil um dos países com mais experiência nessa técnica, se apresentando como alternativa eficaz no tratamento do retinoblastoma infantil avançado, minimizando a necessidade de enucleação (INCA, 2019).

Para essa técnica utilizam-se fármacos pertencentes à quimioterapia clássica, caracterizada por três classes terapêuticas: agentes Alquilantes, Platinas e Inibidor da topoisomerase. O melfalano é um antineoplásico que atua como um agente alquilante/ mostarda nitrogenada derivada da mecloretamina, interferindo com o cruzamento das cadeias de DNA, impedindo a replicação e levando à morte celular, agente não específico de fase do ciclo celular (MOC BRASIL, 2020).

A carboplatina pertencente a uma segunda geração dos análogos de platina atua de forma similar aos agentes alquilantes e apresenta propriedades bioquímicas semelhantes às da cisplatina, produz predominantemente ligações cruzadas intercadeias no DNA, alterando sua estrutura e inibindo sua síntese, agente não específico de fase do ciclo celular (MOC BRASIL, 2020). 
O topotecano possui atividade antitumoral envolvendo a inibição da topoisomerase-I, uma enzima intimamente envolvida na replicação do DNA, aliviando a tensão de torsão introduzida à frente do garfo de replicação em movimento, estabilizando o complexo covalente da enzima com a cadeia clivada do DNA que é um intermediário do mecanismo catalítico, causando a indução de proteínas associadas a quebras em cadeia simples do DNA (EMA, 2009).

O procedimento se baseia na introdução de um microcateter na artéria femoral sendo inserido lentamente através dos vasos sanguíneos até a artéria oftálmica, assim os fármacos são injetados diretamente no vaso sanguíneo que irriga o olho, aumentando a precisão de alcance do alvo desejado proporcionando alta concentração de quimioterápicos no local tumoral, sendo necessária a presença de profissional especializado (PETERSON et al., 2011).

A prestação da assistência ao paciente oncológico inclui diversas especialidades integradas, da qual o profissional farmacêutico desempenha um papel importante devido à alta complexidade do tratamento, sendo fundamentais conhecimentos técnicos específicos, habilidades e competências que visam à promoção da efetividade e segurança do tratamento (ANDRADE, 2009; MATILE, 2008; GEUM, 2016; INCA; REDE CÂNCER, 2019).

\section{METODOLOGIA}

Trata-se de um artigo de revisão da literatura científica com busca nas bases de dados (PubMed, SciELO, Clinical Trials e Biblioteca Cochrane Library) e sites governamentais (INCA, SBOC, Oncoguia, GRAAC, American Cancer Society e Sociedade Mineira de Pediatria).

As palavras chaves para pesquisa foram em português e inglês: Retinoblastoma. Quimioterapia intra-arterial, melfalano, topotecano, carboplatina e seus respectivos correspondentes em inglês, "retinoblastoma", "intraarterial chemotherapy", "melphalan", "topotecan", "carboplatin". 
O período da busca foi de maio/2019 a fevereiro/2020.

\section{DISCUSSÃO}

O retinoblastoma é considerado um câncer grave em que o tratamento requer o uso de técnicas e antineoplásicos específicos (como o melfalano, a carboplatina e o topotecano) em relação à quimioterapia intra-arterial em que a segurança e efetividade necessitam de avaliação (INSTITUTO ONCOGUIA, 2018).

\subsection{MELFALANO}

O agente quimioterápico mais utilizado nessa técnica é o melfalano, devido sua segurança e toxicidade sistêmica mínima. A forma injetável contém $50 \mathrm{mg}$ de substância liofilizada, com $10 \mathrm{~mL}$ de solução diluente. Derivado da mecloretamina, o melfalano interfere no cruzamento das cadeias de DNA, impedindo a replicação e levando à morte celular, agente não específico de fase do ciclo celular (ABRAMSON, 2008; ANVISA, 2018).

Apesar de não haver um protocolo como referência de dosagem, a dose eficaz e segura oftalmológica é menor que $0,5 \mathrm{mg} / \mathrm{kg}$; sua absorção sistêmica é mínima e toxicidade apresentada como neutropenia insignificante. É recomendado antes do procedimento, filtrar o melfalano, pois pode haver pequenas partículas que embolizam os vasos oculares ocasionando complicações no procedimento (MOC BRASIL, 2020).

Uma análise realizada na base de dados internacional Clinical Trials, apontou que existem pesquisas em andamento a fim de comprovar a segurança do fármaco melfalano na terapia intra-arterial. Três estudos foram encontrados em fase de teste e um estudo finalizado (ABRAMSON, 2008; NIH US, 2013; NIH US, 2017; DUANGNATE et al., 2019).

Em novembro de 2017, foi publicado um estudo realizado em Maryland - EUA, a fim de apontar a segurança da técnica intra-arterial quando utilizado o melfalano, objetivando encontrar a dose tolerável do fármaco. Utilizou-se melfalano através da 
artéria femoral, infundido por 30 minutos em 10 crianças com retinoblastoma avançado (fase V), já com indicação de enucleação. Os resultados obtidos apontaram que a técnica apresenta efeitos colaterais sistêmicos e local mínimos (neutropenia de grau 3). Dos 10 olhos, 7 foram recuperados, 1 foi mal sucedido devido a anomalia vascular na artéria, 1 desenvolveu isquemia da retina e 1 desenvolveu retinopatia semelhante à radiação após a braquiterapia. Esta técnica foi descrita pela primeira vez por David H. Abramson no ensaio clínico de fase I / II realizado no Hospital Memorial Sloan-Kettering Câncer Center de Nova York, com o uso do melfalano na quimioterapia intra-arterial na artéria oftálmica para o tratamento de retinoblastoma intraocular (ABRAMSON, 2008).

Outros estudos ainda estão em andamento. Na Espanha um ensaio clínico de fase II não randomizado, visa preservar a acuidade visual dos pacientes utilizando melfalano na terapia intra-arterial em 5 pacientes (NIH US, 2013).

O grupo de oncologia infantil dos EUA dirige um ensaio clínico com 14 participantes, a fim de estimar a taxa de recuperação ocular após o tratamento com melfalano intraarterial em crianças com retinoblastoma unilateral recentemente diagnosticado com doença do grupo D. Este grupo denominado Children's Oncology Group (COG) tratase de uma organização exclusivamente dedicada á pesquisa de câncer na infância e adolescência, possui como membros hospitais infantis, universidades e centros de câncer na América do Norte (NIH US, 2017).

$\mathrm{Na}$ Califórnia, em São Francisco, segue em andamento um estudo a fim de testar a segurança da combinação da terapia quimioterapêutica padrão, alternada e quimioterapia intra-arterial utilizando o melfalano em combinação com outros antineoplásicos (carboplatina, etoposídeo e vincristina) em diferentes esquemas de intervalo. $O$ estudo utilizou 6 pacientes com retinoblastoma intraocular avançado e recém diagnosticado (NIH US, 2017).

Estudos realizados comprovam a eficácia do procedimento da terapia intra-arterial. Uma pesquisa realizada na Tailândia, conduzida de janeiro de 2009 a novembro de 2017, demonstrou resultados positivos, onde se utiliza como principal droga 
o melfalano. Dos 27 olhos de 26 pacientes com retinoblastoma, 7 (26\%) tiveram quimioterapia intra-arterial como tratamento primário e 20 (74\%) tiveram IAC (intra arterial chemotherapy) como tratamento secundário. Os olhos foram categorizados através da Classificação Internacional de Retinoblastoma (ICRB) como grupo B ( $\mathrm{n}=$ 3, 11\%), grupo $C(n=1,4 \%)$, grupo $D(n=12,44 \%)$ e grupo $E(n=11,41 \%)$. O número médio de sessões do IAC foi de 3 (intervalo de 1 a 7). Em um seguimento médio de 32 meses (variação de 3 a 95 meses), a taxa global de recuperação do globo foi de $52 \%$, com $100 \%$ nos grupos B e C, $75 \%$ no grupo D e $9 \%$ no grupo E. Complicações da CIA incluíam vasculopatia oclusiva $(n=4,15 \%)$, hemorragia vítrea ( $n=3,11 \%)$, precipitação da artéria retiniana $(n=2,7 \%)$, estrabismo $(n=2,7 \%)$ e isquêmico transitório ataque $(n=1,4 \%) .(n=25)$ (DUANGNATE, 2019).

\subsection{CARBOPLATINA}

A carboplatina é intensamente distribuída sem ligações às proteínas, sendo o metabolismo hepático e a excreção renal. A administração e diluição ocorrem via endovenosa (EV) em 30 a 120 minutos, intraperitoneal. A diluição é em SF ou SG 5\% (preferencialmente) à concentração de 0,5 a $2 \mathrm{mg} / \mathrm{mL}$, não necessitando de ajuste para a função hepática e, para a função renal a fórmula da AUC (área sob a curva) corrige automaticamente a dose (MOC BRASIL, 2020).

Através de pesquisas registradas no site Clinical Trials só foi encontrado 1 estudo para o tratamento do retinoblastoma com o uso da carboplatina por meio da quimioterapia intra-arterial. Esse estudo teve início em 6 de março de 2019, no Memorial Sloan Kettering Cancer Center, Nova York, Estados Unidos, estando na fase de recrutamento de voluntários (NIH US, 2019).

O objetivo deste estudo é determinar se o tratamento com a carboplatina intra-arterial causa perda auditiva em crianças. Para a realização do estudo foi estabelecido o método observacional de Coorte, com a participação estimada de 50 voluntários de ambos os sexos, portadores da doença, na faixa etária de 3 meses a 18 anos. Os resultados terão uma avaliação auditiva pós intra-arterial (IA) com carboplatina 
permitida somente após 9 meses ou aproximadamente um ano do início do tratamento. A conclusão do estudo está prevista para março de 2021 (NIH US, 2019).

De acordo com o estudo realizado no Japão por especialistas na quimioterapia intraarterial, o uso do medicamento carboplatina que é um derivado da platina, apresenta menos efeitos colaterais que o seu precursor cisplatina, ou seja, menor toxicidade renal, neurológica e gastrintestinal (MANJANDAVIDA et al., 2019).

No entanto, a carboplatina, através de um protocolo é usada com dois medicamentos para a quimioterapia intra-arterial (IAC) unilateral, juntamente com melfalano e topotecano. Já na IAC bilateral, para evitar a toxicidade cumulativa do melfalano que leva a mielossupressão, a dose de melfalano é reduzida com a adição de carboplatina sem comprometer o efeito da IAC. A dosagem recomendada é de 15 a $30 \mathrm{mg}$ (MANJANDAVIDA et al., 2019).

\subsection{TOPOTECANO}

Os dados pré-clínicos sobre a farmacologia ocular de topotecano intra-arterial superseletivo (SSOAI) foram com base em experimentos em um modelo suíno. Após a administração de $1 \mathrm{mg}$ de topotecano SSOAI durante 30 minutos, o topotecano alcançou concentrações vítreas acima do IC50 calculado até 4 horas, uma proporção em cinco vezes maior no plasma vítreo se comparada ao do Melfalano, o agente mais comumente usados para SSOAI. Além de ser encontrado no humor vítreo até 16 horas após a infusão, em contraste com a rápida deterioração do Melfalano na mesma técnica aplicada (SOUZA et al., 2019).

A dose ideal de topotecano por essa via permanece a ser estabelecido, mas que tudo indica que a dose estará entre $0,5 \mathrm{mg}$ a $4 \mathrm{mg}$. Portanto, o topotecano é um interessante medicamento candidato para SSOAI com base na penetração e residência favorável no vítreo (SOUZA et al., 2019).

Devido à alta taxa de exposição vítrea ao plasma do topotecano tal situação pode promover uma penetração favorável do fármaco através da barreira sangue-retiniana, 
e assim atingir camadas mais profundas da região óptica. Estudos preliminares sugerem que nesta estrutura interna do olho a depuração do topotecano é limitada ao vítreo tendo a sua eliminação através da lágrima, com isso, relaciona-se com a menor toxicidade do fármaco em regiões sistêmicas (SCHAIQUEVICH et al., 2014).

Questões favoráveis ao uso oftalmológico estão relacionadas à estabilidade em soluções com diluição, evitando a formação de precipitados que podem ser prejudiciais ao paciente. E a farmacocinética plasmática que associou concomitantemente o topotecano intra-arterial e Melfalano, onde não houve prejuízo clínico ao tratamento (SCHAIQUEVICH et al., 2014).

Desde Novembro de 2011, tem um estudo em andamento, registrado na base de dados Clinical Trials, com 36 pacientes de ambos os sexos na faixa etária de até 15 anos, com histórico de retinoblastoma bilateral recorrente e/ou refratário, que tiveram a administração de topotecano por via intra-arterial oftálmica com o intuito de verificar a eficácia e a segurança. A intervenção se deu pela infusão de topotecano por 30 minutos, a cada 21 dias, por um período de até 18 semanas. Os resultados terão como parâmetros a acuidade visual, eletrorretinograma, potencial evocado visual e ressonância magnética funcional (NIH US, 2017).

Como dito anteriormente, se trata de um estudo em andamento, e por esse motivo, não estão disponíveis dados conclusivos, pois a última atualização desde estudo data de 1 de março de 2017. Portanto, é difícil o estabelecer o papel do topotecano como agente único para o tratamento do retinoblastoma. Mas devido a sua baixa toxicidade ocular, por todas as vias de administração, é provável que o seu lugar no arsenal terapêutico contra o retinoblastoma, se encontre em esquemas quimioterápicos multiagentes combinados (NIH US, 2017).

\section{CONCLUSÃO}

A segurança e efetividade dos fármacos estudados comprovaram que o Melfalano é o principal fármaco utilizado e que atende essas características. O Melfalano pode ser usado individualmente ou em conjunto em doses inferior a $0,5 \mathrm{mg} / \mathrm{kg}$, variando de 
acordo com o tamanho e gravidade do tumor intraocular, tendo seus efeitos adversos comprovado nas pesquisas como leve neutropenia.

Como visto, atualmente há estudos em várias fases que avaliam a efetividade e segurança de outros fármacos, como a Carboplatina e o Topotecano, para serem aplicados individualmente ou em esquema quimioterápico associado em pacientes pediátricos.

Por tratarem de estudos recentes não estão disponíveis até o momento resultados conclusivos, mas o que tudo indica que futuramente esses fármacos, farão parte do arsenal terapêutico, seja individualmente ou fazendo parte em esquemas quimioterápicos multiagentes combinados.

\section{REFERÊNCIAS}

ABRAMSON, David et al. Estudo de fase I / Il da quimioterapia intra-arterial direta (artéria oftálmica) com melfalano para resultados iniciais de retinoblastoma intra-ocular. Nova York, EUA, Volume 115, Edição 8, p.1398-1404.E1, 14 de março de 2008.

Disponível

em:

$<$ https://pubmed.ncbi.nlm.nih.gov/18342944/?dopt=Abstract>. Acesso em: 20 Jun. 2019.

AGÊNCIA EUROPEIA DE MEDICAMENTOS (EMA). ANEXO I RESUMO DAS

CARACTERÍSTICAS DO MEDICAMENTO: Topotecano. EUROPA, 2009.

AMERICAN CANCER SOCIETY. Principais Estatísticas do Retinoblastoma. 2018.

AMERICAN CANCER SOCIETY. Quimioterapia para

retinoblastoma: Quimioterapia intra-arterial. 2018.

AMERICAN CANCER SOCIETY. Terapia a laser (fotocoagulação ou termoterapia) para retinoblastoma. 2018. 
ANDRADE, Cinthya. Farmácia Hospitalar: FARMACÊUTICO EM ONCOLOGIA: INTERFACES ADMINISTRATIVAS E CLÍNICAS. Comissão de Farmácia Hospitalar do Conselho Federal de Farmácia, 2009.

ANVISA, bulário eletrônico. ALKERAN® (melfalana), 2018.

DEPARTAMENTO CIENTÍFICO DE ONCOHEMATOLOGIA. Teste do olhinho e o RETINOBLASTOMA. 2015.

DUANGNATE, Rojanaporn et al. Quimioterapia Intra-Arterial para Retinoblastoma: Experiência de 8 anos de um Instituto de Referência Terciária na Tailândia. Filadélfia, Pensilvânia, Volume 8, Edição 3, p. 211-217, Maio-junho 2019.

ERWENNE, Clélia. ATUALIZAÇÃO CONTINUADA: Os métodos de tratamento e as rotinas em tumores intraoculares. São Paulo, 61(5), Outubro/1998.

FABIAN, Ido et al. Tratamento focal a laser, além de quimioterapia para retinoblastoma. Reino Unido, 07 junho 2017. Disponível em: <https://www.cochranelibrary.com/cdsr/doi/10.1002/14651858.CD012366.pub2/full>. Acesso em: 21 Nov. 2019.

GEUM. Atuação do farmacêutico hospitalar na oncologia: Performance of hospital pharmacist in oncology. Piauí, v. 7, n. 1, p. 54-63, jan/mar 2016. Disponível em: <https://revistas.ufpi.br/index.php/geum/article/view/4018/2916>. Acesso em: 21Out. 2019.

INSTITUTO NACIONAL DE CÂNCER. Educação: Os múltiplos papéis do farmacêutico na atenção oncológica. Rede Câncer, p. 24-29.

INCA - Instituto Nacional de Câncer. QUIMIOTERAPIA INTRA-ARTERIAL PRESERVA OLHO E VISÃO EM TUMOR OCULAR COMUM NA INFÂNCIA: Direto no alvo. p. 11-13, Rede Câncer.

INSTITUTO ONCOGUIA. Quimioterapia para Retinoblastoma. 2017. 
INSTITUTO ONCOGUIA. Diagnóstico do Retinoblastoma. 2017.

INSTITUTO ONCOGUIA. Tipos de Câncer RETINOBLASTOMA: Estadiamento do Retinoblastoma. 2017.

KRONBAUER, Fernando et al. 0 uso da quimioterapia no tratamento do retinoblastoma: Avaliação retrospectiva: The use of chemotherapy in the treatment of retinoblastoma: Retrospective analysis. Porto Alegre (RS), 63(6), Dezembro/2000.

LIANG, Ting et al. Quimioterapia intra-arterial combinada e melfalano intravítreo para o tratamento de retinoblastoma unilateral avançado. China, 13(2): 257-262, 18/fevereiro/2020.

Disponível

em:

https://www.ncbi.nlm.nih.gov/pmc/articles/PMC7013797/>. Acesso em: 10 Fev. 2020.

MANJANDAVIDA, Fairooz et al. Quimioterapia intra-arterial no retinoblastoma Uma mudança de paradigma. Filadélfia, EUA, Volume 67, Edição 6, Página: 740754, 24/Maio/2019. Disponível em: <http://www.ijo.in/article.asp?issn=0301 4738; ;ear=2019; ;olume =67;issue =6; ;page $=740$; epage $=754$; aulast=Manjandavida $>$. Acesso em: 20 Jun. 2019.

MATILE, Elisa. Papel do Farmacêutico na Oncologia: da manipulação à assistência farmacêutica: The Role of the Pharmacist in Oncology: drug manipulation and pharmaceutical support. Curitiba, Vol. 5, N. 14 , p. 29-31, Mai/Ago 2008.

MOC Brasil: Manual de Oncologia Clínica do Brasil. CARBOPLATINA: (Platamine CS $\AA$, Carboplatina genérico). 2020.

MOC Brasil: Manual de Oncologia Clínica do Brasil. MELFALANA: (Alkeran®).2020.

NETO, Joaquim. Diagnóstico Precoce do Retinoblastoma: Boletim Científico Oncologia. 2016. Sociedade Mineira de Pediatria - SMP. Minas Gerais. 
NIH US. National Library of Medicine - Clinical Trails. Quimioterapia Intra-arterial com Melfalan para o Tratamento de Retinoblastoma (RTB) em Estágio Intraocular Avançado. Espanha, 05/2013. Clinical Trails. Disponível em: $<$ https://clinicaltrials.gov/ct2/show/NCT01393769?term=melfalano\&cond=Retinoblast oma\&draw=2\&rank=1>. Acesso em: 11 Fev. 2020.

NIH US. National Library of Medicine - Clinical Trails. Melfalano intra-arterial no tratamento de pacientes mais jovens com retinoblastoma unilateral. Califórnia, EUA, $2017 . \quad$ Disponível em: $<$ https://clinicaltrials.gov/ct2/show/NCT02097134?term=NCT02097134\&draw=2\&ran $k=1>$. Acesso em: 11 Fev. 2020.

NIH US. National Library of Medicine - Clinical Trails. Quimioterapia sistêmica alternada e quimioterapia intra-arterial do melfalano em crianças com retinoblastoma intraocular. Califórnia, São Francisco, 2017. Disponível em: $<$ https://clinicaltrials.gov/ct2/show/NCT02116959?term=NCT02116959\&draw=2\&ran $k=1>$. Acesso em: 11 Fev. 2020.

NIH US. National Library of Medicine - Clinical Trails. Determinando se a carboplatina intra-arterial causa perda auditiva em crianças. Nova York, EUA, 2019. Disponível em: $<$ https://clinicaltrials.gov/ct2/show/NCT03866460?term=NCT03866460\&draw=2\&ran $k=1>$. Acesso em: 12 Fev. 2020.

NIH US. National Library of Medicine - Clinical Trails. Estudo da infusão de topotecano da artéria intra-oftálmica para o tratamento de retinoblastoma (IARB1). 2017. Disponível

em: <https://clinicaltrials.gov/ct2/show/NCT01466855?term=01466855\&draw=2\&rank=1>. Acesso em: 6 Fev. 2020.

ORESTES-CARDOSO, Silvana et al. Epidemiologia das Perdas do Globo Ocular por Retinoblastoma: Eyeball Loss Epidemiology by Retinoblastoma. Camaragibe/PE, v.11, n.2, p. 33-44, abril/junho 2011. 
PETERSON, Eric et al. Infusão seletiva de quimioterapia na artéria oftálmica para retinoblastoma intra-ocular avançado: experiência inicial com 17 tumores. Miami, EUA, Volume 114, Edição 6, junho/2011. Disponível em: $<$ https://pubmed.ncbi.nlm.nih.gov/21294621/>. Acesso em: 20 Out. 2019

PINA, Susana et al. Retinoblastoma: A nossa experiência. In: CONGRESSO PORTUGUÊS DE OFTALMOLOGIA, 55. Lisboa, 2012.

REVISTA DA SOCIEDADE PORTUGUESA DE OFTALMOLOGIA. 10 anos de experiência no tratamento do retinoblastoma. Lisboa, Volume 39, № 2, p. 97-102, Abril-junho 2015.2 Disponível em: $<$ https://repositorio.hff.minsaude.pt/bitstream/10400.10/1559/1/Oftalmologia\%2C\%20 39\%2C\%2097-102.pdf>. Acesso em: 15 Fev. 2020.

RODRIGUES, Karla; LATORRE, Maria do Rosário; CAMARGO, Beatriz de. Atraso diagnóstico do retinoblastoma. Porto Alegre, volume 80, ํㅡ 6, Novembro/Dezembro 2004. Disponível em: <http://www.scielo.br/scielo.php?script=sci_arttext\&pid=S002175572004000800014>. Acesso em: 15 Jul. 2019.

SELISTRE, Simone. Caracterização de pacientes com diagnóstico de retinoblastoma identificados nos Serviços de Oncologia Pediátrica, Oftalmologia e Genética no Hospital de Clínicas de Porto Alegre/RS. Porto Alegre (RS), 114 f., 2013.

SCHAIQUEVICH, Paula et al. Farmacologia ocular do topotecano e sua atividade no retinoblastoma. Buenos Aires, Argentina, Volume 34, Edição 9 - p. 1719-1727, Setembro 2014. Disponível em: <https://www.ncbi.nlm.nih.gov/pubmed/25099219>. Acesso em: 6 Fev. 2020.

SOUZA, Izabella et al. Análise das diferentes abordagens da quimioterapia no tratamento de retinoblastoma. Anápolis, volume 7, suplemento 1, 2019. Disponível em:

$<$ http://revistas.unievangelica.com.br/index.php/educacaoemsaude/article/download/ 3769/2617/>. Acesso em: 6 Fev. 2020. 
Enviado: Agosto, 2020.

Aprovado: Outubro, 2020. 